\title{
CONTAMINACIÓN DEL AIRE A FILO DE CALLE EN QUITO, CASO ESTUDIO GUAYAQUIL Y ESPEJO
}

\section{Air Pollution at Edge of Street in Quito, Case Study Guayaquil and Espejo}

\author{
${ }^{1,2}$ David Chuquer Solá*, ${ }^{1}$ Santiago Ampudia Vásquez, ${ }^{1}$ Carolina Puertas De La Cruz, \\ ${ }^{1}$ Leonardo Bustamante Granda, ${ }^{3}$ Carlos Reina Velasco, \\ ${ }^{2}$ Francisco Ramírez Cevallos*
}

${ }^{1}$ Facultad de Ciencias Exactas y Naturales. Pontificia Universidad Católica del Ecuador. Av. 12 de octubre 1076 y Roca, Quito, Ecuador. Código postal 170525. ${ }^{2}$ Facultad de Arquitectura, Diseño y Arte. Pontificia Universidad Católica del Ecuador. Av. 12 de octubre 1076 y Roca, Quito, Ecuador. Código postal 170525. ${ }^{3}$ Facultad de Ciencias Químicas, Universidad Central del Ecuador. Francisco Viteri y Gilberto Sobral s/n, Ciudad Universitaria, Quito, Ecuador. Código postal 170521.

*dchuquer295@puce.edu.ec,dschuquer@uce.edu.ec

\section{$R$ esumen}

Se estudió la contaminación del aire a filo de calle en el Centro Histórico de Quito (DMQ) en un punto de alto flujo vehicular mediante el monitoreo continuo de gases y material particulado entre el $5 \mathrm{y}$ 12 de abril de 2018. Se obtuvieron los perfiles horarios de las concentraciones de contaminantes y se pudo explicar su comportamiento. Las mediciones en el estudio no sobrepasaron los límites permisibles nacionales ni internacionales de calidad del aire; sin embargo se observaron picos anómalos en el caso del dióxido de azufre. Se evaluó la correlación cruzada de las series de tiempo entre los datos reportados por estaciones regionales de la Secretaria de Ambiente del DMQ y los datos obtenidos en este estudio, hallándose que el monóxido de carbono presenta una mayor concentración a filo de calle. Al realizar el análisis de componentes principales (ACP) se determinó varios contaminantes correlacionados, lo que corroboran sus ciclos de formación y demuestran la influencia de factores meteorológicos en la contaminación de aire a filo de calle. Finalmente se determinó que las concentraciones de $\mathrm{PM}_{10}$ en aire interior son mayores a lo registrado en aire exterior en el sitio de muestreo.

Palabras claves: contaminación del aire, Quito, contaminantes criterio, ACP, correlacion cruzada.

\section{A bstract}

Air pollution at the street edge was studied in the Historical Downtown of Quito (DMQ) at a point of high vehicular flow by continuous gas monitoring and particulate material between April 5 and 12, 2018. Time profiles were obtained of the pollutant concentrations and their behavior was explained. The measurements in the study did not exceed the national and international permissible limits of air quality, however abnormal peaks were observed in the case of sulfur dioxide. The cross-correlation of the time series between the data reported by regional stations of the Secretary of Environment of the DMQ and the data obtained in this study was evaluated, finding that carbon monoxide presents a higher concentration on the street edge. When performing the main components analysis (PCA), several correlated pollutants were determined, which corroborates their formation cycles and de- 
monstrates the influence of meteorological factors on air pollution at the street edge. Finally, it was determined that the concentrations of $\mathrm{PM}_{10}$ in indoor air are higher than that registered in outdoor air in the sampled point.

Keywords: p-Nitrophenol; photocatalytic degradation; Activated carbon; titanium dioxide; composite

\section{INTRODUCCIÓN}

La contaminación atmosférica es el principal riesgo ambiental para la salud de las personas (1). Según la Organización Mundial de la Salud (OMS), el $92 \%$ de personas en el mundo vive en lugares donde no se cumplen los niveles de contaminantes criterio recomendados por esta entidad en lo que se refiere aire ambiente. En el 2012 se estimaron 93000 muertes en Centro y Sudamérica relacionadas con el decremento en la calidad del aire, siendo las enfermedades más recurrentes el derrame cerebral, las enfermedades coronarias y el cáncer de pulmón. Durante el mismo año se estimó que en el Ecuador se produjeron 2008 muertes relacionadas con la mala calidad del aire debido principalmente a enfermedades coronarias (2), es por ello que el control de la contaminación del aire se ha convertido en un eje principal de la agenda de entidades gubernamentales en el mundo (3). Uno de los problemas más graves es la toxicidad de las partículas la cual ha sido corroborada por diversos estudios a nivel celular los cuales demuestran que afectan profundamente la salud provocando estrés oxidativo en las células, respuestas inmunológicas, inflamación sistémica de órganos respiratorios, daños genómicos del ADN que conlleva a la formación potencial de mutaciones y finalmente tumores(4,5). Las principales fuentes de contaminantes que afectan la calidad del aire son el escape de los autos, la resuspensión de partículas y actividades de cocción de alimentos en locales comerciales (6). En el Distrito Metropolitano de Quito (DMQ) existen alrededor de 488000 vehículos (7) que utilizan como combustible gasolina de alto y bajo octanaje (entre 92 y 86 RON) y diésel con un contenido de azufre de $500 \mathrm{ppm}(8)$, que al estar a $2850 \mathrm{msnm}$ provocan su combustión incompleta favoreciendo la emisión de hidrocarburos aromáticos policíclicos (9). Según reportes de la Secretaria de Ambiente del DMQ, las mayores emisiones de gases y partículas se ubican entre las $7 \mathrm{~h} 00$ y $10 \mathrm{~h} 00$ am y entre las $17 \mathrm{~h} 00$ y $20 \mathrm{~h} 00 \mathrm{pm}$ (10).

Sobre la base de la evidencia científica, desde el 2004 la Secretaria de Ambiente del DMQ controla la calidad del aire mediante una Red Automática (RAUTO) con ocho estaciones regionales a lo largo de la ciudad y los valles aledaños con monitores continuos que entregan información horaria de los niveles de contaminantes en la ciudad. Así mismo, dispone de la Red de Monitoreo Pasivo (REMPA) que cuenta con 39 dispositivos para la supervisión de la calidad del aire a filo de calle, cuyos valores indican la concentración promedio de contaminantes en periodos más extensos (días o meses), utilizando estimaciones respecto a la adsorción de contaminantes en los dispositivos. A pesar de la aplicabilidad de los monitores pasivos (11), lamentablemente no permiten obtener perfiles horarios del comportamiento de los contaminantes. La RAUTO ha reportado que en el DMQ durante el 2015 y 2016 se han superado los límites máximos permisibles recomendados por la OMS en materia de partículas PM10, PM2.5 y O 3 (12), mientras que la REMPA ha reportado que existen mediciones a filo de calle donde los contaminantes superan los límites permisibles como en la Marín, Necochea, Basílica, Puente del Guambra, Cumbayá, Camal, Carapungo y Centro Histórico (12). Numerosas investigaciones han resaltado la necesidad de realizar estudios de contaminación del aire a filo de calle debido a las diferencias encontradas respecto a los niveles de contaminantes registrados por estaciones de medición regional (13-15), por lo que se hace necesario un estudio profundo a filo de calle con monitores continuos que permitan obtener perfiles horarios del comportamien- 


\section{Perfíles}

Revista Científica

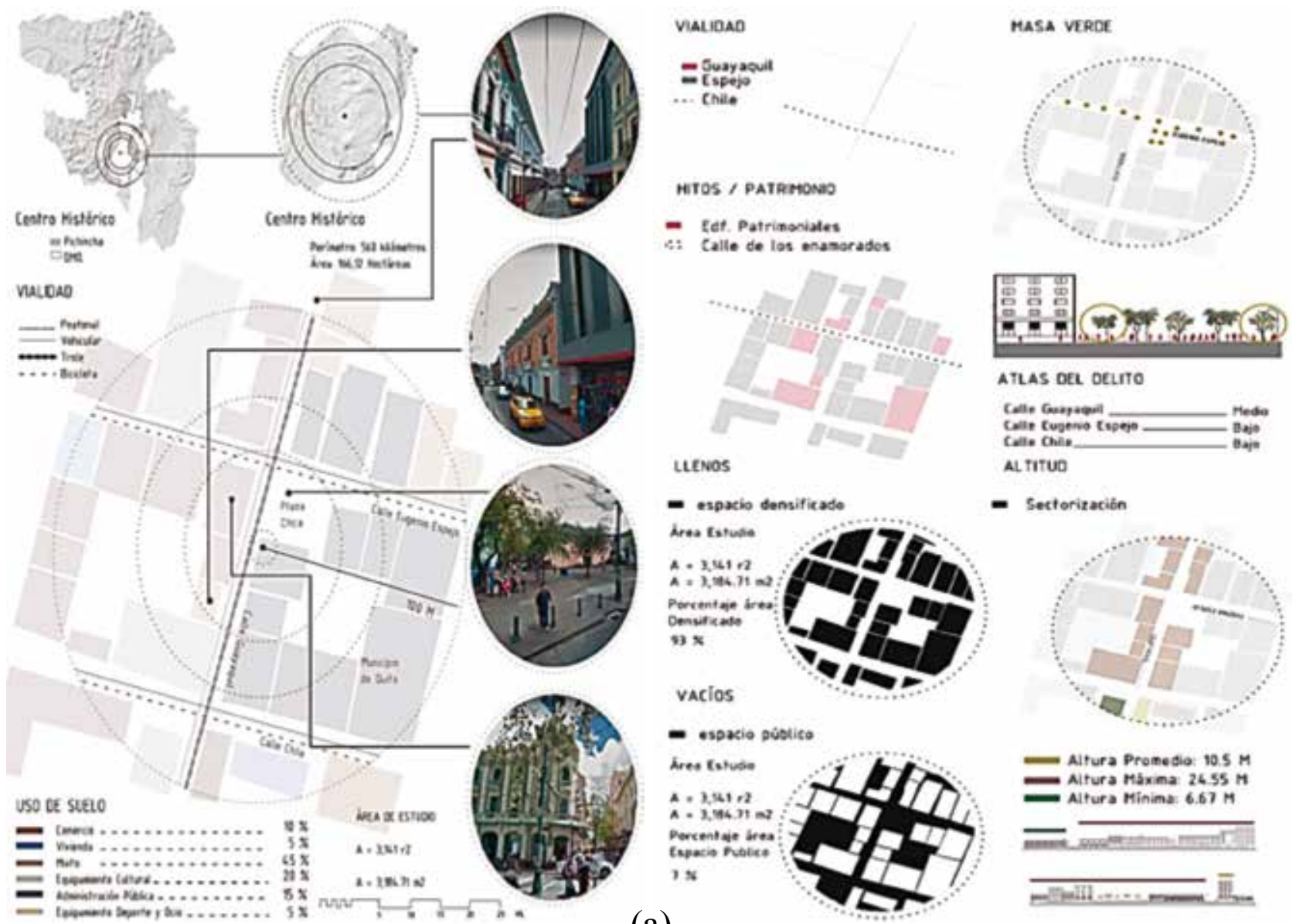

(a)
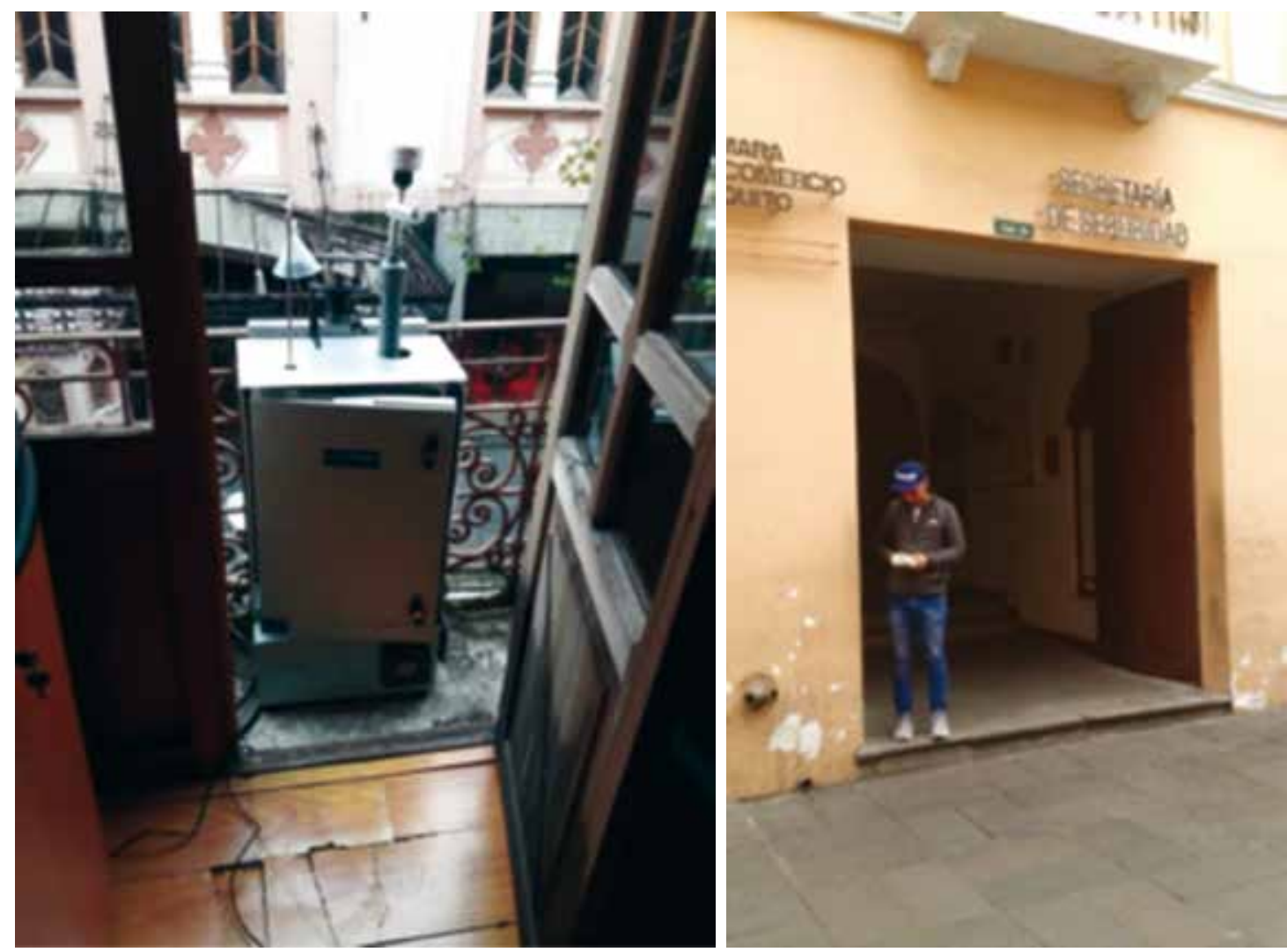

(b)

Figura 1. a. Descripción del punto de monitoreo Secretaria de Seguridad y Gobernabilidad del DMQ; b. Puntos de muestreo de calidad del aire 
to de los contaminantes atmosféricos ya que aproximadamente el $25 \%$ de la población en el DMQ vive en cercanías de vías con alto tráfico vehicular (16).

Dentro de los objetivos del estudio está conocer el estado de la contaminación del aire a filo de calle en el Centro Histórico de Quito, obtener perfiles horarios para las concentraciones de los contaminantes, comparar los valores obtenidos con límites máximos permisibles nacionales e internacionales de calidad del aire y realizar el análisis de componentes principales para identificar las variables que están correlacionadas en la contaminación del aire a filo de calle en el punto de monitoreo. En este caso se presentan resultados del monitoreo realizado en la Secretaría de Seguridad y Gobernabilidad del DMQ, ubicada en el centro histórico de Quito en la calle Espejo, entre Guayaquil y Montúfar como lo indica la figura 1. El trabajo fue presentado como ponencia en el "I Congreso Internacional de Química Ecuador 2018" el 31 de julio de 2018 y se enmarca en el proyecto "Estudio de la contaminación del aire a filo de calle y sus impactos en zonas de alto tráfico de la ciudad de Quito" financiado por la Pontificia Universidad Católica del Ecuador (PUCE), en el que actualmente se están realizado estudios similares al expuesto en este trabajo en 10 zonas críticas de la ciudad.

\section{MATERIAL Y MÉTODOS}

Se ejecutó el monitoreo de calidad del aire en la Secretaría de Seguridad y Gobernabilidad del DMQ ubicada en la calle Espejo entre Montúfar y Guayaquil en el Centro Histórico del DMQ en uno de los puntos de mayor congestión vehicular de la ciudad. Algunas características sociales y arquitectónicas de la zona se especifican en la figura 1, siendo una zona de alta densidad poblacional con calles estrechas que puede provocar la acumulación de contaminantes. Se realizaron mediciones de aire ambiente en exteriores, en el balcón del edificio de la Secretaría de Seguridad y Gobernabilidad, punto de coordenadas UTM $777017.04 \mathrm{~m} \mathrm{E}, 9975583.79 \mathrm{~m} \mathrm{~S}$, zona $17 \mathrm{M}$. Las mediciones de aire interior se realizaron en el ingreso al edificio, en coordenadas UTM $777109.25 \mathrm{~m} \mathrm{E}$, 9975589.55m S, zona 17M.

La calidad del aire ambiente fue monitoreada con el AQM60 Environmental Monitor V5.0 de AEROQUAL durante 7 días por 24 horas entre el 5 y 12 de abril de 2018. La calidad del aire interior a nivel del suelo fue monitoreada con el equipo AEROCET 531S de METONE durante el 09 y 10 de abril de 2018 por 12 horas entre las $07 \mathrm{~h} 00$ y las $19 \mathrm{~h} 00$. Los parámetros analizados, y los métodos instrumentales se detallan en la tabla 1 .

Los datos de partículas $\left(\mathrm{PM}_{0 \mathrm{E}}, \mathrm{PM}_{1}, \mathrm{PM}_{2.5}, \mathrm{PM}_{4}\right.$ y $\left.\mathrm{PM}_{10}\right)$ fueron reportados en microgramos por metro cúbico $(\mu \mathrm{g} /$ $\mathrm{m}^{3}$ ) corregidos a condiciones de referencia, es decir a 25 grados Celsius $\left({ }^{\circ} \mathrm{C}\right)$ y 760 milímetros de mercurio
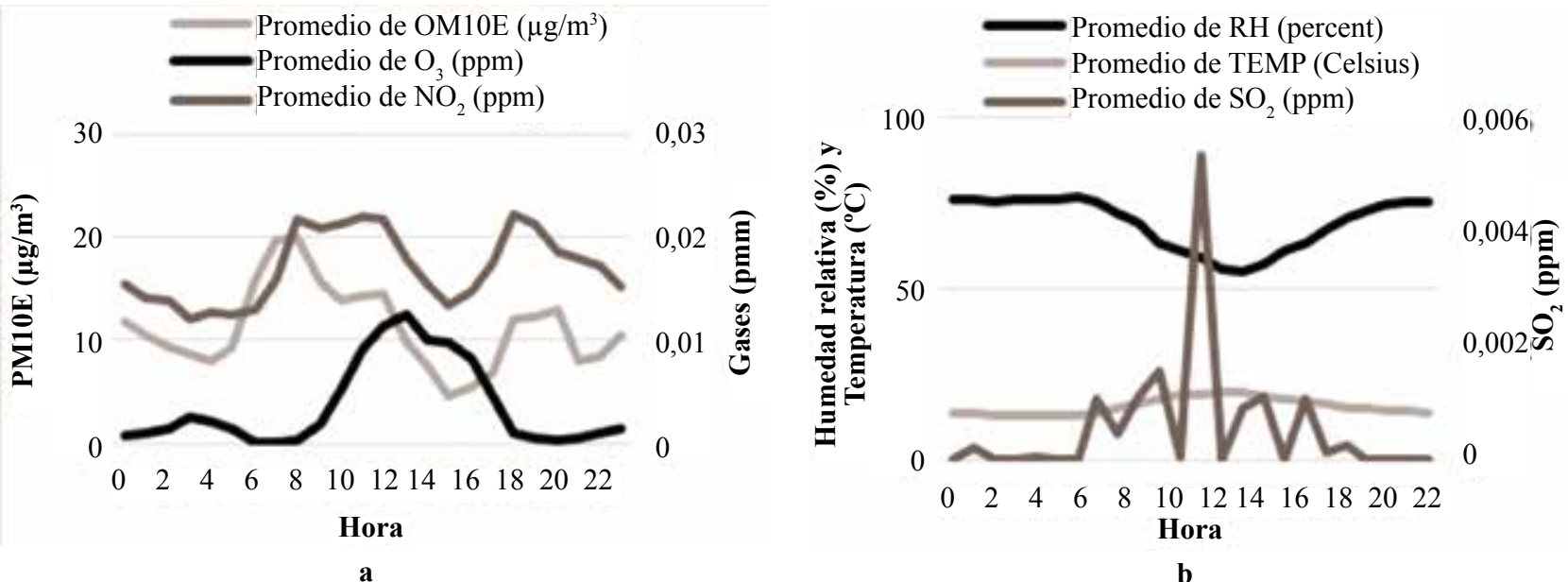

Figura 2. a. Series de tiempo con promedios de una hora para $\mathrm{O} 3, \mathrm{NO}_{2}$ y PM10E; b. Series de tiempo con promedios de una hora para $\mathrm{SO}_{2}$ con $\mathrm{RH}$ y TEMP. 


\begin{tabular}{|c|c|c|c|}
\hline Sensor & $\begin{array}{c}\text { Método } \\
\text { instrumental }\end{array}$ & Aplicación & Captura de datos \\
\hline Monóxido de carbono (CO) & $\begin{array}{l}\text { Semiconductor sensible } \\
\text { a gas (GSS) }\end{array}$ & Aire exterior & Cada dos minutos \\
\hline Dióxido de carbono $\left(\mathrm{CO}_{2}\right)$ & $\begin{array}{l}\text { Infrarrojo no dispersivo } \\
\text { (NDIR) }\end{array}$ & Aire exterior & Cada dos minutos \\
\hline Ozono $\left(\mathrm{O}_{3}\right)$ & GSS & Aire exterior & Cada dos minutos \\
\hline Dióxido de nitrógeno $\left(\mathrm{NO}_{2}\right)$ & GSS & Aire exterior & Cada dos minutos \\
\hline Dióxido de Azufre $\left(\mathrm{SO}_{2}\right)$ & GSS & Aire exterior & Cada dos minutos \\
\hline $\begin{array}{c}\text { Partículas con diámetro } \\
\text { aerodinámico menor a } 10 \\
\text { micrómetros en exteriores } \\
\text { (PM10E) }\end{array}$ & Disperción de luz & Aire exterior & Cada dos minutos \\
\hline $\begin{array}{l}\text { Velocidad del viento (WS) y } \\
\text { dirección del viendo (WD) }\end{array}$ & Sensor ultrasónico & Aire exterior & Cada dos minutos \\
\hline Temperatura (TEMP) & $\begin{array}{l}\text { Sensor bnd-gab } \\
\text { de temperatura }\end{array}$ & Aire exterior & Cada dos minutos \\
\hline Humedad (RH) & Sensor de film capacitivo & Aire exterior & Cada dos minutos \\
\hline $\begin{array}{c}\text { Partículas en interiores PM1, } \\
\text { PM2.5, PM4, PM10 y partí- } \\
\text { culas sedimentables totales } \\
\text { (TSP) }\end{array}$ & Contador de partículas & Aire interior & Cada diez minutos \\
\hline
\end{tabular}

Tabla 1: Descripción de monitores de calidad de aire y captura de datos

(mmHg) según lo establecido en el Texto Unificado de Legislación Secundaria del Ministerio del Ambiente del Ecuador (17), mientras que los gases $\left(\mathrm{CO}, \mathrm{CO}_{2}, \mathrm{O}_{3}, \mathrm{NO}_{2}\right.$, $\mathrm{SO}_{2}$ ) se reportaron en partes por millón ( $\mathrm{ppm}$ ). Debido a que la normativa nacional de calidad del aire indica límites permisibles en $\mu \mathrm{g} / \mathrm{m}^{3}$ a condiciones de referencia, para compararlos con los valores medidos en el estudio, se aplicó la siguiente ecuación:

$$
\mu \mathrm{g} / \mathrm{m}^{3}=1000 * \mathrm{ppm}^{*} \mathrm{~K}
$$

Donde $\mathrm{K}$ es un factor relacionado con la densidad del contaminante a condiciones de $25^{\circ} \mathrm{C}$ y $760 \mathrm{mmHg}$. Los factores son: $\mathrm{K}_{\mathrm{CO}}=1.14 ; \mathrm{K}_{\mathrm{O} 3}=1,96 ; \mathrm{KN}_{\mathrm{O} 2}=1,88 ; \mathrm{KS}_{\mathrm{O} 2}=2,62$.

Se realizaron series de tiempo con promedios de una hora de los datos adquiridos por los equipos detallados anteriormente. El análisis de componentes principales (ACP) se realizó con el complemento de Excel, XLSTAT ${ }^{\circledR}$ que genera un círculo de correlación de las variables analizadas. La comparación de las series de tiempo obtenidas en los monitoreos realizados en este estudio con los datos reportados por la Secretaría de Ambiente del DMQ (estación regional Centro Histórico para los parámetros $\mathrm{NO}_{2}, \mathrm{CO}, \mathrm{O}_{3}$ y $\mathrm{SO}_{2}$ ) se realizó aplicando la correlación cruzada con el paquete estadístico Rstudio ${ }^{\circledR}$ Version 0.99 .903 , obteniéndose los gráficos que relacionan la función de autocorrelación con el número de retrasos de cada una de las series de tiempo evaluadas (18).

\section{RESULTADOS}

En las figuras 2 y 3 se detallan las series de tiempo de los contaminantes analizados y su comparación en diferentes contextos como el ámbito meteorológico o su correlación con otros contaminantes criterio. La figura 4 detalla los resultados del análisis de componentes principales.

\section{DISCUSIÓN}

\section{Análisis de series de tiempo}

La figura 2a indica el comportamiento del $\mathrm{NO}_{2}, \mathrm{O}_{3}$ y $\mathrm{PM}_{10 \mathrm{E}}$ durante el día en promedios de una hora tomando en cuenta las mediciones de los siete días de monitoreo (entre el 5 y 12 de abril de 2018). Los patrones observados son recurrentes en varias zonas de Quito (19). 


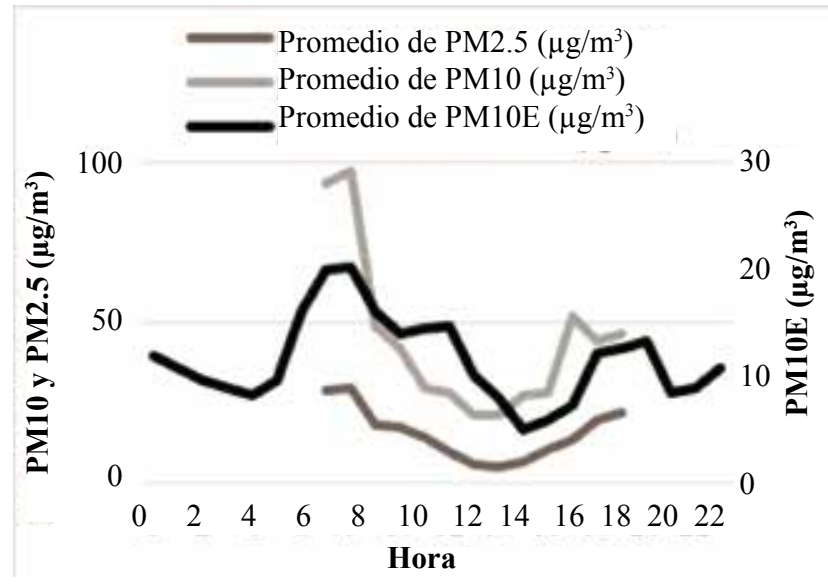

a

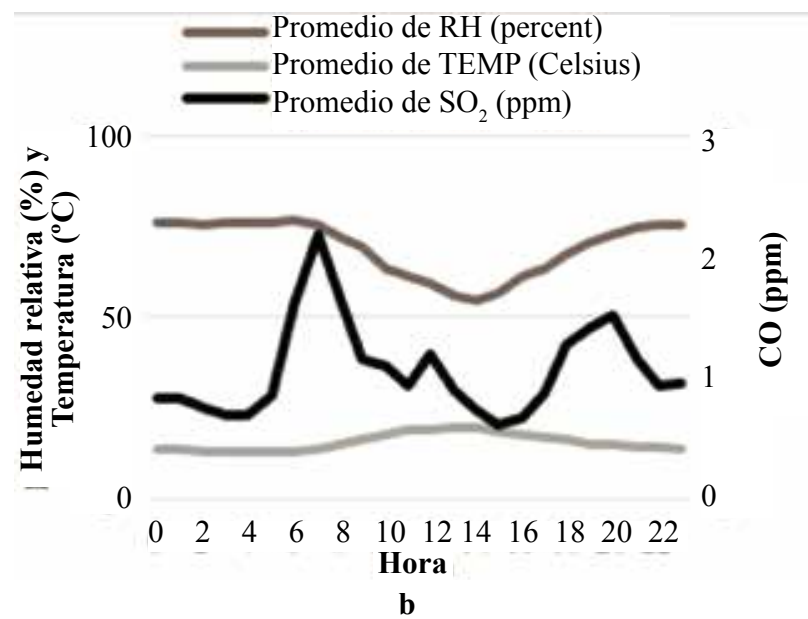

Figura 3. Series de tiempo con valores de una hora para: a. material particulado en exteriores $\mathrm{PM}_{10 \mathrm{E}}$ e interiores $\mathrm{PM}_{10}$ y $\mathrm{PM}_{2.5}$; b. CO y la influencia de TEMP y RH.

El PM10E registra picos máximos entre las $6 \mathrm{~h} 00$ y $8 \mathrm{~h} 00$, debido principalmente al alto tráfico vehicular de la mañana y las bajas temperaturas que no permiten la adecuada dispersión de los contaminantes. A medida que avanza el día y la altura de la capa de mezcla atmosférica aumenta (incluso superando los 2000 metros sobre el nivel del suelo), las concentraciones de los contaminantes disminuyen (20). Entre las $18 \mathrm{~h} 00$ y $20 \mathrm{~h} 00$ se registra nuevamente un pico por el aumento del tráfico vehicular en la zona.
El comportamiento de las partículas durante el día en parte se debe a que el aumento de la humedad relativa en las primeras horas de la mañana y en la tarde provoca una disminución en la eficiencia de combustión de motores favoreciendo el aumento de emisión de partículas (21) lo cual es corroborado por un aumento en las concentraciones de $\mathrm{CO}$ y $\mathrm{NO}_{2}$ en las horas pico. Se ha evidenciado que la composición química de las partículas en zonas en las que existe mayor tráfico vehicular contienen mayor cantidad de metales pesados como $\mathrm{Pb}$, As, y $\mathrm{Zn}$ respecto a zonas con bajo flujo vehicular (16).

La intensa actividad antropogénica, acompañada por la alta radiación solar de la ciudad (máxima a las 13h00)
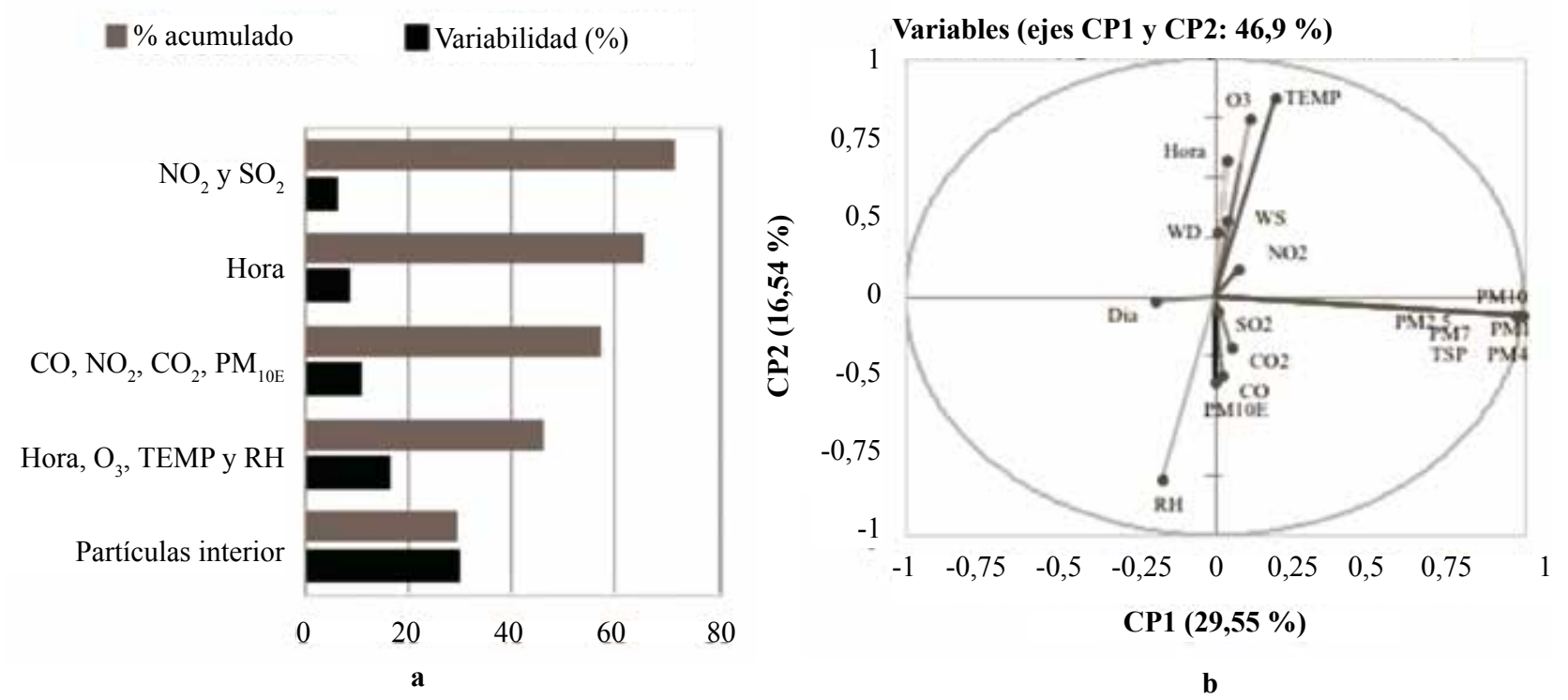

Figura 4. a. Contribución de las componentes principales a la variabilidad total de los datos; b. Circulo de correlación de variables medidas 
provoca condiciones químicas que favorecen la formación $\mathrm{O}_{3}$ que registra un pico máximo a las $13 \mathrm{~h} 00$, mientras que el $\mathrm{NO}_{2}$ registra picos máximos entre las 8 y 12 del mañana y a las $18 \mathrm{~h} 00$. Es notorio que los niveles de $\mathrm{NO}_{2}$ permanecen estables durante el periodo de máxima actividad antropogénica ( $7 \mathrm{~h} 00$ a 19h00) excepto durante los picos de $\mathrm{O}_{3}$ por su reducción a monóxido de nitrógeno. En la figura $2 \mathrm{~b}$, se observa que el $\mathrm{SO}_{2}$ es un parámetro dependiente de los niveles de humedad relativa en el ambiente debido a su alta constante de Henry respecto a los otros contaminantes criterio (22). El $\mathrm{SO}_{2}$ aumenta su concentración considerablemente en periodos del día cuando la humedad disminuye y la temperatura aumenta, es decir entre las $10 \mathrm{~h} 00$ y $15 \mathrm{~h} 00$.

En el caso del $\mathrm{CO}$, se observan picos de concentración a las $7 \mathrm{~h} 00$ y $20 \mathrm{~h} 00$, periodo en el que se registra la mayor actividad vehicular como se observa en la figura $3 \mathrm{~b}$ similar a lo que ocurre con el $\mathrm{PM}_{10 \mathrm{E}}$. Parámetros meteorológicos como la humedad relativa y la temperatura no influyen drásticamente en los niveles de este contaminante.

\section{Comparación con límites permisibles}

Durante el monitoreo se registraron picos de concentración que exceden el valor numérico de los límites permisibles de las normas nacionales e internacionales vigentes, sin embargo al realizar los promedios temporales no se registraron incumplimientos de la normativa respecto a los límites permisibles establecidos en el Texto Unificado de Legislación Secundaria del Ministerio del Ambiente (TULSMA) Libro VI anexo 4 ni los límites recomendados para calidad del aire establecidos por la OMS $(17,23)$.

\section{Correlación cruzada}

Se compararon mediante correlación cruzada las series de tiempo de los parámetros $\mathrm{CO}, \mathrm{NO}_{2}, \mathrm{SO}_{2}$ y $\mathrm{O}_{3}$ entre la estación regional "Centro Histórico" y la estación a filo de calle instalada en este estudio. Los resultados sugieren que las concentraciones de $\mathrm{CO}$ registradas a filo de calle son superiores y con comportamientos similares a lo reportado por la estación Centro Histórico de la Secretaría de Ambiente. Para el caso del $\mathrm{O}_{3}$ y $\mathrm{NO}_{2}$, los valores medidos en este estudio son similares a la estación de monitoreo regional (Centro Histórico), mientras que el $\mathrm{SO}_{2}$ es un parámetro netamente dependiente del punto monitoreo en estudio. No se pudo comparar los niveles de partículas $\mathrm{PM}_{10 \mathrm{E}}$ con los niveles reportados por una estación regional debido a que no existen datos reportados por la Secretaría de Ambiente del DMQ para estaciones cercanas.

\section{Comparación aire interior y exterior}

La Agencia de Protección Ambiental de los Estados Unidos ha indicado que las intersecciones señalizadas de tráfico y carreteras son sitios donde usualmente existen concentraciones de contaminantes atmosféricos superiores respecto a lo registrado en estaciones regionales. Las emisiones son principalmente de los escapes de vehículos (24) pudiendo existir ingreso de estos contaminantes a los interiores vía ventilación e infiltración (25). La intrusión de contaminantes afecta tanto a residencias como oficinas y entidades educativas (8). Estudios sobre la afectación que sufren niños que viven en las cercanías de vías con alto tráfico en Quito $(<$ a $100 \mathrm{~m})$ confirman un decremento en su salud cardiovascular $(16,26)$. Con este antecedente, se comparó la concentración de partículas en exteriores e interiores en el punto de monitoreo estudiado cuyos comportamientos son muy similares entre sí como se observa en la figura 3a; sin embargo las concentraciones de partículas en interiores son mayores a las registradas en exteriores. Estos resultados son consistente a lo publicado por Raysoni (8).

\section{ACP}

Finalmente se realizó el ACP de las variables analizadas. La figura 4 a muestra los pesos de cada uno de las componentes principales (CP) en la variabilidad total de los datos, observándose que los primeras dos CP explican el 46,09\% de la variabilidad. La primera $\mathrm{CP}$ aborda las partículas medidas en interiores y la segunda CP la variabilidad de los datos a través del día, los niveles de ozono, la humedad y la temperatura. Respecto al círculo de correlación descrita en la figura $4 \mathrm{~b}$, podemos determinar la formación de grupos de variables que se com- 
portan de manera similar. Un grupo de variables correlacionadas es el formado por las partículas medidas en aire interior. Otro grupo de variables correlacionadas son los contaminantes primarios $\mathrm{CO}, \mathrm{CO}_{2}$ y $\mathrm{PM}_{10 \mathrm{E}}$ los cuales están inversamente correlacionados con los niveles de $\mathrm{O}_{3}$ y TEMP.

\section{CONCLUSIONES}

El estudio permitió conocer el comportamiento de varios contaminantes criterio en un punto de alto tráfico del DMQ. Pudieron obtenerse los perfiles horarios de los contaminantes en el periodo de análisis e identificar la influencia de factores meteorológicos en los niveles de los contaminantes y sus correlaciones. Se determinó que los niveles registrados en el punto de monitoreo no sobrepasaron los máximos permisibles establecidos por la legislación ecuatoriana de calidad del aire ni los lineamientos de la OMS; sin embargo, se encontró que a filo de calle existen niveles superiores de $\mathrm{CO}$ respecto a lo reportados por las estaciones regionales. Adicionalmente, mediante mediciones realizadas en ambientes interiores se pudo encontrar que existen mayores concentraciones de partículas respecto al aire exterior. Actualmente estudios similares al desarrollado en este trabajo se están realizando en diez zonas críticas de la ciudad lo que va a permitir tener un panorama integral de las condiciones de calidad de aire ambiente e interior en zonas afectadas por las vías de alto tráfico vehicular.

\section{AGRADECIMIENTOS}

Los autores agradecen a la Pontifica Universidad Católica del Ecuador por el financiamiento a través del proyecto O13024. Adicionalmente los autores agradecen a la Dra. Astrid Aguirre de la Secretaría de Seguridad y Gobernabilidad del DMQ por las gestiones para el acceso a los puntos de monitoreo.

\section{eferencias}

1. Prüss-Ustün, A., Wolf, J., Corvalan, C., Bos, R., Neira, M. Preventing disease through healthy environments: a global assessment of the burden of disease from environmental risks [Internet]. 2da ed. Geneva: World Health Organization; 2016 [citado 21 de agosto de 2018]. Disponible en: http:// www.who.int/quantifying_ehimpacts/publications/preventing-disease/en/

2. WHO. Ambient air pollution: A global assessment of exposure and burden of disease [Internet]. Geneva: WHO; 2016. 1-131 p. Disponible en: http://www.who.int/phe/publications/air-pollution-global-assessment/en/

3. 52 Consejo Directivo de OPS. Plan Estratégico de la Organización Panamericana de la Salud 2014-2019. «En pro de la salud: Desarrollo sostenible y equidad» [Internet]. Washington: OPS; 2013 oct [citado 21 de agosto de 2018] p. 1-162. Report No.: OD345. Disponible en: http://apps.who.int/ iris/handle/10665/165204

4. Steiner S, Bisig C, Petri-Fink A, Rothen-Rutishauser B. Diesel exhaust: current knowledge of adverse effects and underlying cellular mechanisms. Archives of Toxicology. 1 de julio de 2016;90(7):1541-53.

5. Cevallos VM, Díaz V, Sirois CM. Particulate matter air pollution from the city of Quito, Ecuador, activates inflammatory signaling pathways in vitro. Innate Immunity. 2017;23(4):392-400.

6. Lee BP, Li YJ, Yu JZ, Louie PKK, Chan CK. Characteristics of submicron particulate matter at the urban roadside in downtown Hong Kong-Overview of 4 months of continuous high-resolution aerosol mass spectrometer measurements. J Geophys Res Atmos. 18 de junio de 2015;120(14):7040-58.

7. Romero, Daniel. De 488 mil vehículos, 400 mil se matricularon en el 2016 en Quito. El Comercio [Internet]. 12 de enero de 2017 [citado 21 de agosto de 2018]; Disponible en: https://www.elcomercio.com/actualidad/quito-vehiculos-matriculacion-tramites.html 
8. Raysoni AU, Armijos RX, Weigel MM, Montoya T, Eschanique P, Racines M, et al. Assessment of indoor and outdoor PM species at schools and residences in a high-altitude Ecuadorian urban center. Environ Pollut. julio de 2016;214:668-79.

9. Brachtl MV, Durant JL, Perez CP, Oviedo J, Sempertegui F, Naumova EN, et al. Spatial and temporal variations and mobile source emissions of polycyclic aromatic hydrocarbons in Quito, Ecuador. Environ Pollut. 1 de febrero de 2009;157(2):528-36.

10. Secretaria de Ambiente del DMQ. Informe final Inventario de emisiones de contaminantes criterio 2011 [Internet]. Quito: Secretaria de Ambiente del DMQ; 2014 [citado 21 de agosto de 2018] p. 1-53. Report No.: 5to. Disponible en: http://www.quitoambiente.gob.ec/ambiente/index.php/informes\#inventario-de-emisiones-2011

11. Rosario L, Pietro M, Francesco SP. Comparative Analyses of Urban Air Quality Monitoring Systems: Passive Sampling and Continuous Monitoring Stations. Energy Procedia. 1 de noviembre de 2016;101:321-8.

12. Diaz Suarez Valeria. Informe de la calidad de aire - 2016 [Internet]. Quito: Municipio del DMQ; 2017 may p. 1-55. Report No.: IAMQ/17. Disponible en: http://www.quitoambiente.gob.ec/ambiente/images/Secretaria_Ambiente/red_monitoreo/informacion/ICA2016.pdf

13. Moore A, Figliozzi M, Monsere C. Air Quality at Bus Stops. Transp Res Rec. 9 de octubre de 2012;2270:76-86.

14. Wang X (Richard), Oliver Gao H. Exposure to fine particle mass and number concentrations in urban transportation environments of New York City. Transp Res D. 1 de julio de 2011;16(5):384-91. 15. Gulliver J, Briggs DJ. Personal exposure to particulate air pollution in transport microenvironments. Atmos Environ. 1 de enero de 2004;38(1):1-8.

16. Raysoni AU, Armijos RX, Weigel MM, Echanique P, Racines M, Pingitore NE, et al. Evaluation of Sources and Patterns of Elemental Composition of PM2.5 at Three Low-Income Neighborhood Schools and Residences in Quito, Ecuador. International Journal of Environmental Research and Public Health [Internet]. 2017;14(7). Disponible en: http://www.mdpi.com/1660-4601/14/7/674

17. Ministerio del Ambiente del Ecuador. Norma de calidad del aire ambiente o nivel de inmisión, Libro VI Anexo 4. Registro Oficial jun 7, 2011 p. 8-15.

18. Venables, W.N., Ripley, B.D. Modern Applied Statistics with S. 4th ed. New York: Springer-Verlag New York; 2002. 1-498 p. (Statistics and Computing).

19. Cazorla M. Análisis de los datos horarios de radiación solar y abundancia de ozono del Distrito Metropolitano de Quito del 2007 al 2012. Aci. 2013;5(2):c67-78.

20. Cazorla M. Air quality over a populated Andean region: Insights from measurements of ozone, NO, and boundary layer depths. Atmos Pollut Res. 1 de enero de 2016;7(1):66-74.

21. Zalakeviciute R, López-Villada J, Rybarczyk Y. Contrasted Effects of Relative Humidity and Precipitation on Urban PM2.5 Pollution in High Elevation Urban Areas. Sustainability [Internet]. 2018;10(6). Disponible en: http://www.mdpi.com/2071-1050/10/6/2064

22. Sander R. Compilation of Henry's law constants (version 4.0) for water as solvent. Atmos Chem Phys. 2015;15(8):4399-4981.

23. WHO. Air quality guidelines. Global update 2005. Particulate matter, ozone, nitrogen dioxide and sulfur dioxide [Internet]. third revision. Copenhagen: Publications WHO Regional Office for Europe; 2006 [citado 7 de septiembre de 2018]. 484 p. Disponible en: http://www.euro.who.int/_data/ assets/pdf_file/0005/78638/E90038.pdf

24. Goel $\bar{A}$, Kumar P. A review of fundamental drivers governing the emissions, dispersion and exposure to vehicle-emitted nanoparticles at signalised traffic intersections. Atmos Environ. 1 de noviembre de 2014;97:316-31.

25. Ai ZT, Mak CM. A study of interunit dispersion around multistory buildings with single-sided ventilation under different wind directions. Atmos Environ. 1 de mayo de 2014;88:1-13. 
26. Armijos RX, Weigel MM, Myers OB, Li W-W, Racines M, Berwick M. Residential Exposure to Urban Traffic Is Associated with Increased Carotid Intima-Media Thickness in Children. J Environ Public Health. 2015;2015:11. 